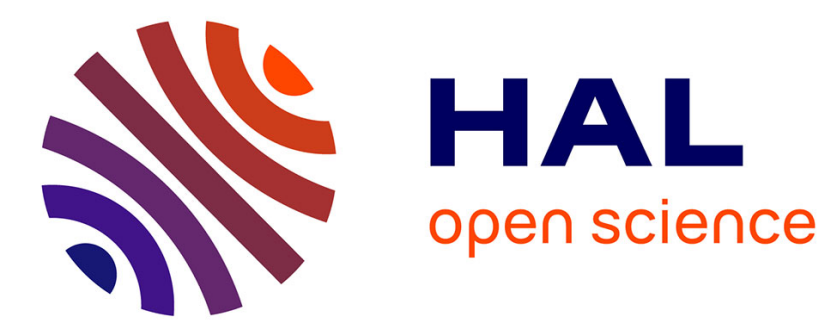

\title{
L'intimité partagée par nécessité : entre respect et liberté
}

Eve Gardien

\section{To cite this version:}

Eve Gardien. L'intimité partagée par nécessité: entre respect et liberté. Corps à cœur - Intimité, amour, sexualité et handicap, Erès, pp.37-57, 2014. hal-01419800

\section{HAL Id: hal-01419800 \\ https://hal.science/hal-01419800}

Submitted on 19 Dec 2016

HAL is a multi-disciplinary open access archive for the deposit and dissemination of scientific research documents, whether they are published or not. The documents may come from teaching and research institutions in France or abroad, or from public or private research centers.
L'archive ouverte pluridisciplinaire HAL, est destinée au dépôt et à la diffusion de documents scientifiques de niveau recherche, publiés ou non, émanant des établissements d'enseignement et de recherche français ou étrangers, des laboratoires publics ou privés. 


\title{
L'intimité partagée par nécessité : entre respect et liberté
}

\author{
Eve Gardien \\ Sociologue \\ MCF Université Rennes2 \\ ESO (UMR 6590)
}

Intervenir auprès d'une personne dépendante pour l'accompagner dans les actes de sa vie quotidienne, a deux conséquences majeures, à savoir: participer au moins partiellement à sa vie privée, entrer journalièrement dans son intimité. Ces conséquences ne sont un choix ni pour l'un, ni pour l'autre protagoniste, mais bien un effet inéluctable de cet accompagnement. Plus avant, l'intimité est usuellement partagée pour raison de survie organique et de bienséance sociale pour la personne handicapée, pour raisons professionnelles ou par engagement personnel pour les autres. Rien qui relève des usages habituels de l'intimité : pas de choix d'apprivoisement réciproque donc, et pas davantage de don et de contre don conçu ou perçu comme tel, simplement une nécessité - la dépendance - faisant force de loi.

La dépendance concernant les besoins vitaux et/ou les actes de la vie quotidienne implique d'être mis journalièrement à nu, au risque d'une atteinte à la pudeur, d'être accompagné dans de nombreuses activités par une aide humaine, au risque d'une publicisation de la vie privée, de voir pénétrer son domicile plusieurs fois par jour, au risque de ne plus être maître chez soi. Ce qui suppose corollairement pour le professionnel de risquer de porter atteinte à autrui en de multiples occasions, et à divers niveaux, mais aussi, d'être soi-même affecté par diverses émotions éprouvés en ces situations extra-ordinaires, par une charge psychique particulière liée à l'attention à autrui, par des effets de résonnance et de résurgence découlant de ses propres expériences de l'intimité. Si les risques ne sont pas identiques, ils sont néanmoins amplement partagés. Une asymétrie cependant : l'intimité n'est pas réciproquement partagée.

Si l'intimité varie dans ses formes et ses actualisations en fonction des aires culturelles, des périodes historiques, des statuts sociaux, elle reste une expérience probablement universelle de la vulnérabilité de chaque être lorsqu'une atteinte à son être-même est possiblement imminente. C'est pourquoi, étant donné l'importance accordée à la personne dans notre culture et nos textes légaux, le partage de l'intimité est usuellement choisi, sous le sceau de la confiance, et non pas contraint. Le consentement est un prérequis. Or, la dépendance dans les actes de la vie quotidienne impose d'expérimenter des modalités interactionnelles inhabituelles, hors des sentiers battus, de vivre des situations extra-ordinaires, de s'exposer à des situations peu conventionnelles. Le partage de l'intimité avec des aides humaines n'est souvent pas un choix, seulement une nécessité, radicale dans ses conséquences.

Ce chapitre est consacré à une description fine des divers impacts de ce partage de l'intimité par nécessité, avec une focale concernant plus particulièrement les incidences pour les personnes dépendantes. Seront ainsi traités plusieurs dimensions de cette expérience: le sentiment d'une dignité en sursis, le trouble émotionnel de l'ordre interactionnel, la publicisation de la vie privée, la possible institutionnalisation de la vie à domicile, l'impact sur l'ordre domestique et la « sédimentation biographique », l'atteinte au déploiement et au développement d'une subjectivité réflexive.

\section{Des intimités variables : essai de définitions pratiques}

L'intime : qu'est-ce à dire ? L'intimité n'est pas une conception de toujours, mais une compréhension datée dans l'histoire humaine. En effet, l'existence de ce vocable est attestée à partir du XIVème siècle. Emprunté au latin intimus, il désigna tout d'abord ce qui est « le plus en dedans, le plus personnel», et se nuança au début du XVlème siècle de l'idée de "vie intérieure, généralement secrète, d'une personne ». Ce terme a initialement été largement employé pour désigner une ou des personnes très liées, voire unies avec une autre, à comprendre au sens de l'amitié aussi bien que des rapports amoureux et/ou charnels. Victor Hugo évoque 
d'ailleurs à ce sujet « ce qui est strictement personnel et généralement tenu caché aux autres, en particulier ce qui se manifeste par un contact charnel » (1821). Par extension, cette qualification d'« intime » relative aux liens unissant étroitement les personnes, sera mobilisée pour nommer ce qui relie fortement aux choses à partir de 1765, pour désigner un lieu ou une atmosphère propice à l'intimité à compter de 1849. L'acception concernant le domaine privé et/ou secret de l'individu sera très valorisée au cours de la période romantique.

S'il est souvent affirmé qu'il existe autant d'intimités que d'individus, il n'en reste pas moins que l'analyse de matériaux issus d'enquêtes sociologiques montre incontestablement quelques dimensions récurrentes des expériences qualifiées d'« intime " par nos contemporains. Si ces dimensions sont rarement évoquées toutes ensemble au cœur de chaque expérience singulière, elles présentent pourtant un noyau de significations recoupant très largement les différentes acceptions étymologiques évoquées précédemment. Ceci nous amène à inférer par conséquent une causalité sociale à l'émergence d'expériences personnelles d'intimité. Autrement-dit, l'expérience toute singulière et personnelle de l'intimité existerait en raison d'une sociabilité et en conséquence d'une socialisation.

Que donne à comprendre l'analyse de matériaux provenant d'enquêtes de terrain ? Certains interviewés privilégient nettement dans leur discours les aspects corporels de l'intimité, allant de la simple nudité, en passant par un sentiment corporel d'être intégralement soi, jusqu'à des échanges affectivo-sexuels variés. D'autres évoquent ce qu'ils ne donnent pas souvent ou même jamais à voir, autrement-dit certaines de leurs pensées, quelques-uns de leurs sentiments. Pour d'autres encore, l'intimité convoque leurs relations de confiance et/ou de grande proximité physique. Ils se rappellent alors leurs proches, amis et compagnons de vie. Les derniers jettent une toute autre lumière sur leur expérience de l'intimité en soulignant l'intensité et la prégnance de leurs liens à des objets, des meubles, des photos, des ambiances ou même à leur domicile, comme autant de supports de souvenirs ou la trace tangible de leur vie singulière.

Ainsi, l'intimité se donne à voir plurielle dans son empirie, variable dans ses expériences. L'individu expérimente des intimités, comme un emboîtement de poupées russes, et cela en fonction des circonstances, des personnes et des situations, ou bien, une intimité à géométrie variable, c'est selon. En outre, l'intimité ne réfère pas concrètement aux mêmes dimensions de l'expérience pour tous, cela suivant les investissements de chacun. Certains s'engagent dans les voies sensuelles, et d'autres davantage dans les tréfonds de leur subjectivité, quelques-uns s'attachent aux indices matériels du passé qui les fondent. Tous ne confèrent pas à l'ensemble de ces dimensions de l'expérience le qualificatif d' « intime », même si ce sont bien ces dimensions qui sont généralement convoquées.

Cette expérience plurielle des intimités concerne tout aussi bien les professionnels, les aidants familiaux que les personnes en situation de handicap. Mentionnons l'existence de caractéristiques particulières à l'intimité au cours de l'enfance, au cours de laquelle elle est en voie de constitution. De fait, l'intimité n'est pas innée. Elle a des causes sociales pour origine, des conditions sociales au maintien de son vécu.

Dans la suite de notre propos, nous viserons principalement à mettre en lumière les enjeux inhérents à la pénétration nécessaire dans l'espace intime d'adultes dépendants et consentants. Ceci permettra néanmoins un éclairage concernant les faits relatifs à l'enfance.

\section{L'intimité pénétrée par nécessité : la dignité en sursis}

L'intimité est une expérience personnelle, vécue présentement, construite sur les bases d'une histoire collective et des socialités passées. Les intimités d'hier ne sont pas celles d'aujourd'hui. De fait, il nous semble important de souligner que notre société contemporaine est parcourue par des courants de détraditionalisation et d'individualisation qui opèrent de concert des transformations profondes de l'intimité. Làencore, un approfondissement aurait été bien venu pour une compréhension plus aboutie des réflexions livrées ici. De plus, la société contemporaine se caractérise par un ensemble de domaines sociaux équivalents et non plus hiérarchisés, l'un ne soumettant plus les autres selon un schéma pyramidal. L'individu se trouve donc face à une liberté plus importante qu'un siècle auparavant, liberté qui se révèle à la fois une chance mais aussi un fardeau, et ce jusque dans les aménagements de l'intimité. Cependant, si la société moderne propose à l'individu une pluralité de rôles, de situations, de contextes sociaux et une relative liberté d'interprétation desdits rôles, la personne dépendante, quant-à-elle, ne se voit pas moins corsetée par un ensemble d'attentes et de préjugés la concernant. 
Ainsi, il va souvent de soi qu'une personne dépendante doive livrer au regard et aux mains d'autrui son intimité corporelle pour diverses activités de soin et d'accompagnement dans sa vie quotidienne. Les circonstances et les conséquences sociales entourant ce dévoilement sont rarement mises en lumière et interrogées. Or, même si l'intervention dans son intimité corporelle est généralement jugée nécessaire par la personne en situation de handicap, elle n'est pas pour autant une formalité ou une banalité. De fait, lorsque la parole est donnée aux usagers de divers services, le constat est fait que, de façon récurrente, certains expriment le désir de ne pas être regardé nu, d'être couvert au cas où autrui viendrait à passer sans prévenir, de ne pas voir leur corps manipulé par une personne de sexe opposé, entre autres exemples...

Pareillement, il est d'usage que la personne dépendante ouvre son domicile aux professionnels, ou encore sa chambre lorsqu'elle est hébergée en institution, cela en raison des nécessités de l'intervention. II n'est pas rare que le professionnel soit en possession des clefs du logement ou encore qu'un interdit porte sur le verrouillage de la porte de la chambre en institution, la nuit notamment. Pourtant, bien que cette pénétration des lieux d'intimité soit assurément utile, cette pratique professionnelle ne s'opère pas sans remous. Là encore, entre utilité consensuelle et mises en pratique, un espace n'est pas interrogé, alors que certaines récriminations ordinaires des personnes dépendantes font précisément état de l'usage d'entrer dans leur chambre sans frapper ni attendre de réponse, ou bien d'arriver à leur domicile comme en terrain conquis.

Pour autant, nulle personne dépendante ne conteste véritablement le principe de cette entrée dans son intimité par autrui. Qu'il s'agisse de professionnels, qu'il s'agisse d'aidants familiaux, l'utilité de l'intervention fait évidence pour tous. De fait, les enjeux au quotidien sont souvent de taille, à savoir : la survie organique, le maintien d'une hygiène et d'une décence corollaire, la présentation de soi à autrui sous une apparence convenable, voire valorisante.

Cependant, cette entrée dans l'intimité par nécessité, autrement-dit hors des cadres sociaux conventionnels, provoque régulièrement de la gêne ou du malaise, parfois des réactions de pudeur ou encore de honte, d'autre fois du déni et de l'indifférence, et bien d'autres ressentis encore. De fait, même lorsque les rituels proxémiques ont été modifiés et intériorisés, bien qu'une certaine accoutumance s'opère toujours avec le temps, les personnes dépendantes n'éprouvent pas ou peu de plaisir à voir leur intimité ainsi pénétrée. Etre dans l'obligation de dévoiler sa nudité ou encore éprouver des difficultés majeures pour préserver de la publicisation des pans entiers de sa vie privée, n'est pas une expérience plaisante. Un vécu d'intrusion est d'ailleurs souvent prégnant, et ce malgré la bienveillance des intervenants, les nécessités consensuelles et les bénéfices secondaires à la situation. Nos repères socioculturels codent cette entrée dans l'intimité d'autrui comme une modalité possible d'atteinte à sa dignité.

Parallèlement, pénétrer l'intimité de l'autre ne va pas toujours de soi pour le professionnel. Les intervenants sont pour la plupart d'entre eux très conscients des réticences que suscite une part de leur activité professionnelle. Ils devinent aisément les raisons de ces tensions, même si elles restent usuellement peu verbalisées. Et tous savent au plus profond d'eux-mêmes que pénétrer l'intimité d'autrui, même avec son consentement, c'est risquer de profaner la personne elle-même.

Ainsi c'est sur un paradoxe que l'accompagnement au quotidien des personnes dépendantes se fonde. L'intervenant est aux prises avec un choix insoluble : respecter la personne ou intervenir dans son intimité. Quant à la personne dépendante, elle fait face à un dilemme non moins cornélien : accepter cette pénétration de son intimité ou préserver sa dignité. L'intériorisation d'autres habitudes proxémiques, les accoutumances sécrétées au long des situations qui se déroulent, ne peuvent complètement dissoudre cette tension : le soin et l'accompagnement dans les gestes du quotidien sont aussi des violations des espaces proxémiques conventionnels, des transgressions des marques de respect usuelles. Par voie de conséquence, nul ne sort indemne de ce paradoxe, et tous sont confrontés à des risques sociaux importants : porter atteinte à autrui ou être blessé au plus profond d'eux-mêmes.

\section{Risque d'atteinte à l'intimité corporelle}

Révéler son intimité corporelle ou pénétrer l'intimité d'autrui, a pour corollaire de devoir affronter un déferlement d'émotions en soi et en l'autre qui peut mettre à tout instant la relation en danger, si l'on n'y prend pas garde. En effet, depuis sa plus tendre enfance, l'être humain a appris progressivement et intériorisé un ensemble de significations, de codes, de conventions, d'usages relatifs au corps. Il a notamment été initié aux méandres de l'intimité corporelle, à sa haute valeur et à sa protection impérative, parfois à certaines de ses 
subtilités telles que les camps nudistes ou les douches dans les vestiaires de sport collectif. Ainsi, l'individu a assimilé et généralement fait sien un rapport au corps culturellement construit, lequel stipule des distances à maintenir, des réalités corporelles à cacher, des actes à retenir. Certains prétendent à la libération des corps. Si chacun a goûté le plaisir d'être nu sous le soleil d'été dans sa plus tendre enfance, aucun ne tenterait cette expérience adulte sur la place du marché, et cela sans que l'usage des forces de police ou la mention de l'aspect illégal dudit comportement ne soient utiles pour l'en retenir. Les corps d'aujourd'hui ne sont pas libérés, mais autrement socialisés.

Par ailleurs, il arrive couramment qu'une personne dépendante, depuis sa naissance ou sa petite enfance, intériorise un autre rapport à sa nudité, du fait même d'une socialisation corporelle différente. Elle est accoutumée à se montrer dévêtue, à ce que son corps soit manipulé par autrui, et ce en raison des diverses interventions journalières nécessaires à la satisfaction de ses besoins. Cela fait-il de cette personne un être humain impudique pour autant ? Force est de constater que, cette socialisation spécifique aidant, l'éprouvé de pudeur est plus rare, parfois inexistant ou encore simplement tu. Cependant, nul doute que des regards salaces, des mains trop insistantes, des postures lascives, des propos licencieux, soient généralement vécus comme humiliants et dégradants. Ainsi, des limites et des conventions relative à l'intimité corporelle ont bien été intériorisées, et ce en adéquation avec la socialisation subie.

Reste donc que, du côté de l'intervenant néophyte ou de la personne récemment devenue dépendante, entrer dans l'intimité d'autrui ou accepter cette entrée, par nécessité, ne fait pas partie des schémas culturels usuellement transmis. Les codes et les conventions manquent au début de cet exercice social périlleux. Qu'est-il bienséant d'éprouver en pareille situation? Quels sont les attendus sociaux ? Comment se comporter? Autant de repères qui ne préexistent pas de par une socialisation classique pour l'intervenant novice ou l'homme récemment devenu dépendant. Ainsi, tant que l'expérience du handicap n'est pas suffisante, ni codifiée, les comportements restent-ils soumis à des tensions importantes, lesquelles ne perdront de leur vivacité qu'avec le temps. Pléthores de comportements d'adaptation sont par ailleurs observables: faire comme si de rien n'était, rester autant qu'il est possible sur un registre strictement professionnel, banaliser ou dédramatiser, détourner le sens de la situation, etc. Autant de façon de se prémunir des risques encourus de part et d'autre, sans qu'il soit possible néanmoins d'éradiquer ces affects redoutables pour l'ordre interactionnel.

De quelles émotions est-il donc question ? Un premier ensemble d'affects concerne la qualité esthétique du corps donné à voir. Le jugement de l'intervenant est souvent appréhendé par la personne dénudée lors des premiers accompagnements, le dévoilement de petits défauts jusqu'alors cachés sous ses vêtements est également redouté. Parallèlement, l'aidant peut être inquiet des interprétations posées sur le moindre de ses regards ou de ses mots, troublé à l'idée de pouvoir être considéré comme un voyeur, en souci de blesser autrui sans l'avoir souhaité.

D'autres ressentis semblent prendre couramment une place importante lors de cette entrée dans l'intimité corporelle par nécessité. II s'agit du possible désir sexuel. La sempiternelle question du pouvoir de séduction est bien évidemment couramment posée, que l'autre attire ou pas. De là, découle l'inquiétude réciproque de devoir supporter et gérer les avances d'autrui, ses gestes et son toucher, son odeur et ses regards. A l'inverse, ce peut être la peur de ne pas être à la hauteur, d'être rejeté, ou encore de laisser l'autre de marbre, qui peut rendre difficiles les échanges, là encore pour les deux parties. Pour d'autres, plus pudiques, il s'agira d'arriver à cacher ses émotions et ses désirs, de ne pas embarrasser autrui. D'autres encore chercheront à maîtriser les manifestations d'un corps incontrôlable, exposant des signaux sans lien aucun avec leurs affects, et laissant autrui supposer ce qui n'est pas...

Viennent aussi les émotions marquées du sceau de la différence corporelle. Leur intensité peut varier avec l'étendue de l'expérience de la dépendance. Par exemple, pour quelques-uns, il peut être insupportable de se rappeler leur impuissance, une avalanche de colère ou de dépression les ensevelit alors. Pour d'autres, la crainte de croiser un regard condescendant ou méprisant, de supporter une vision de soi réductrice, est insoutenable. Dans le même temps, l'inquiétude de rappeler à l'autre ce qu'il n'est plus, de raviver des souvenirs douloureux par sa seule présence valide, peut submerger certains intervenants. D'autres sont au bord de l'épuisement face au flot sans fin des gestes à effectuer, des besoins à satisfaire. Quelques-uns vivent l'inquiétude de produire un geste qui se révèlera douloureux, une parole manquant de tact, etc.

Enfin, lorsque les barrières protectrices et si fragiles ont été franchies, intentionnellement ou pas, la crainte et 
la peur s'installent. Parfois pour une partie, parfois pour les deux, l'un redoutant une possible récidive, l'autre appréhendant une dénonciation tardive. Un labyrinthe d'émotions pour un dédale de complexité effrayée, dont il est toujours difficile de s'extirper par la suite.

\section{Risque d'atteinte à la vie privée}

La vie privée appartient au domaine plus vaste de la vie quotidienne. Cette sous-catégorie présente des caractéristiques spécifiques en termes d'usages et de conventions sociales. En effet, dans le cadre de la vie privée, il est d'usage de faire valoir ses préférences et affinités relationnelles, de fabriquer un cocon de sociabilités à sa convenance, alors que dans l'espace public chacun a droit de cité. Toutefois, cet égard socialement accordé aux liens de sympathie et d'attirance dans le cadre de la vie privée, se trouve conventionnellement limité. Par exemple, on choisit rarement sa belle-famille. Cependant, il est attendu de la civilité de part et d'autre, davantage si affinité.

Pareillement, autre distinction séparant la vie privée de la vie publique: les codes de conduites, les conventions en matière de communication, les usages sociaux. Ils sont généralement plus souples, ou, tout du moins, différents. Ainsi, ces rituels de communication signifient davantage la proximité des personnes, la connivence, l'affection ou l'attachement. Ils sont aujourd'hui moins structurés autour de la déférence aux statuts de chacun, parents ou enfants, mais davantage conditionnés par une prise en compte de la singularité de chacun. Cet espace social autorise également des comportements dits de « régression ».

Ces mêmes rituels, ces mêmes sentiments et échanges s'estompent généralement dès que des individus extérieurs à la vie privée participent à l'échange social en cours. Par exemple, un homme peut se lover comme un enfant dans les bras de sa femme, scène qu'il ne produira jamais devant de simples voisins ou des collègues de travail. Pareillement, une femme ou un homme peut battre régulièrement son partenaire, et s'en abstenir devant autrui. Ainsi, l'espace de la vie privée permet des échanges sociaux particuliers, parce que protégés des regards extérieurs et des jugements du tout-venant. La vie privée est donc corrélée avec un espace de liberté pour l'individu, à l'intérieur duquel il peut davantage faire valoir ses choix, ses principes, ses envies, ses goûts, ses impulsions, etc., sans être soumis à un contrôle social trop contraignant et élargi.

La vie privée se distingue donc de la vie publique notamment par une adaptation comportementale des individus qui ne donnent pas à voir d'eux la même chose. C'est un certain relâchement du contrôle sur son apparence qui caractérise les sociabilités au cœur de la vie privée, et, a contrario, une maîtrise plus aboutie des apparences qui marque les interactions dans l'espace public. Un signe marquant l'entrée dans la sphère des échanges privatifs est la possibilité que s'octroient alors les acteurs en présence de paraître sous leur plus mauvais jour, de laisser transparaître leurs humeurs maussades, d'exprimer leurs opinions politiques avec virulence, mais aussi de partager un fou rire. Alors que des relations afférant à la vie publique, révèleront bien souvent les mêmes acteurs sous des jours plus pondérés, plus retenus dans l'expression de leurs affects, plus policés, sauf s'ils savent leurs affects et opinions largement partagés par les autres interactants en présence.

La vie privée est finalement un espace social où l'acteur peut s'autoriser des écarts à la bienséance, contredire sciemment ses discours énoncés et ses comportements adoptés au sein de l'espace public, donner à voir des faits ou des qualités généralement dissimulés, sans que cela offusque autrui. Bien au contraire, c'est cette liberté et ce sont notamment ces connivences qui soudent les liens entre intimes.

Or que dire des liens sociaux unissant l'intervenant et la personne dépendante ? S'ils ne sont pas intimes pourtant de nombreux aspects de leurs interactions pourraient le laisser supposer... En effet, le professionnel est journalièrement confronté à la difficulté de respecter la vie privée de la personne dépendante alors qu'il pénètre son domicile ou sa chambre, participe de fait à de nombreux évènements de sa vie privée, et glane par conséquent bien involontairement souvent des informations ne le concernant pas, ni lui ni sa pratique. Si son intervention est strictement à caractère professionnelle, elle est pourtant nécessairement colorée par la vie privée et domestique de la personne.

Plus avant, sa simple présence introduit un tiers extérieur dans la vie privée de la personne, qui plus est un tiers qui a rarement été choisi, avec lequel il n'y a pas toujours affinité. Il en découle une restriction tacite, mais importante, des possibles au sein de l'espace privé, voir une impossibilité d'exprimer ouvertement des sentiments ou des opinions, de se comporter de telle ou telle façon durant tout le temps de l'intervention du professionnel. Les individus s'adaptent souvent spontanément au mode de sociabilité en public, dès que la 
présence de celui qui est vécu comme intrus est repérée. II n'est pas toujours besoin d'un règlement intérieur ou du rappel du « cadre » pour ramener à de la sociabilité publique un espace d'échanges privés. Sur la base de ces constats, une question se pose : cet espace de liberté qu'est la vie privée peut-il le rester en situation d'intimité partagée par nécessité ? En effet, comment préserver sa vie familiale lorsque l'appel téléphonique du proviseur du collège de l'enfant résonne au beau milieu de la douche matinale? Comment garder confidentielle pour quelques temps encore une relation amoureuse naissante sans demander à l'être aimé de se restreindre à des rencontres entre $9 \mathrm{~h}$ et $11 \mathrm{~h} 30$ ? Comment dissimuler ses ennuis avec le fisc ou encore cacher sa consommation de stupéfiants ? Comment garder pour soi ses pratiques religieuses, sa volonté de lire journalièrement la Bible ou le Coran ? Autant de dilemmes auxquels sont confrontées au quotidien les personnes dépendantes, et qui mettent directement en cause la préservation d'une image de soi ou d'une réputation.

La pénétration de l'intimité par nécessité réduit donc souvent drastiquement, voire annihile parfois, la possibilité même d'une vie privée pour la personne dépendante. Ainsi, ces interventions nécessaires sont là encore fondées sur un paradoxe: compenser le handicap ou préserver la vie privée de la personne dépendante ? De fait, ces deux principes, essentiels pour le moins, ne sont pas toujours compatibles dans une pratique concrète.

\section{Risque d'institutionnalisation du domicile}

Le domicile, le chez-soi renvoie étymologiquement à l'endroit où l'on est maître, maître des lieux, mais aussi, pour le père, maître de la famille, des domestiques ou encore des esclaves. Le domicile renvoie donc explicitement au lieu d'exercice de sa puissance en tant que "maître », en tant que lieu privé et donc exceptionnellement soumis à des interventions directes de l'Etat. Le domicile est par suite le lieu par excellence où la personne a le sentiment d'être souveraine, d'exercer un droit d'usage sur un territoire qui est le sien, et ce dans la durée, en toute légalité.

En outre, une autre signification du terme « domicile » émerge à l'analyse étymologique, à savoir : l'abri. En ce sens, le domicile doit aussi être compris comme un espace géographique délimitant bien plus qu'un milieu caractérisé par ses extériorités, ses limites ou ses frontières. Le domicile permet de circonscrire un intérieur d'un extérieur, permettant alors de concevoir un lieu de vie où l'individu étend son empire, sans avoir à ferrailler avec les contingences et les contraintes du dehors, sans avoir à batailler avec les influences externes. Le domicile est le lieu que l'on peut rendre inaccessible aux autres, un lieu privatisable, un espace qui ne serait pas public. Ce domicile apparaît alors comme un chez soi, lequel, de part sa continuité et sa prévisibilité, est favorable à un sentiment de sécurité et de protection, et engendre par voie de conséquence une forme de réassurance. Le domicile est donc le lieu du repos et de la tranquillité. Le domicile est l'espace social qui accueille tout particulièrement la vie privée, les liens d'affection et d'attachement.

Le domicile, tout à la fois lieu d'exercice de son empire et lieu de refuge, est un espace où celui qui y vit, entasse des objets personnels ainsi que de nombreux souvenirs, des meubles et des photos souvent, donne libre cours à ses goûts en matière de décoration et de confort, déploie progressivement son art de vivre. En ce sens, le domicile accueille progressivement les nombreuses strates matérielles découlant de la vie de la personne, est le témoin mais aussi le réceptacle d'une trajectoire de vie, et subit un processus de « sédimentation de la biographie» de l'habitant en son sein même. C'est pourquoi le premier appartement est bien souvent plus nu que celui de la fin de vie. C'est aussi pourquoi déménager peut devenir traumatisant: plus cet entassement de strates matérielles témoins est important, investi affectivement, et plus les personnes sont attachées à leur domicile. Ce lieu est alors, de leurs points de vue, façonné à leur image. Attenter à ce territoire labouré par les nombreux sillons que l'individu a creusé par ses habitudes, ses usages du domicile et ses déplacements, autrement-dit par sa manière d'habiter, pénétrer ce lieu par effraction ou sans accord préalable, c'est porter atteinte à la personne elle-même. Le domicile renvoie alors au chez-soi comme fort intérieur, comme lieu de préservation d'une histoire personnelle et interpersonnelle, à partir duquel chacun étaye sa subjectivité et son identité propres.

Quel risque une personne en situation de handicap peut-elle courir à son propre domicile ? Celui que l'intervention nécessaire au regard de sa dépendance, ne corresponde pas à ses attentes et devienne un outil d'assujettissement du sujet par un processus d'institutionnalisation du domicile. Qu'est-ce à dire ? De fait, l'intervention à domicile n'est pas toujours un choix de la personne directement concernée mais peut se 
révéler être celui de son environnement humain, ou bien un choix fait par défaut, ou encore une option prise en faveur du moins pire, faut-il le rappeler : par nécessité. II est vrai que l'intervention à domicile est souvent une condition sine qua non de la vie ou du maintien chez soi, il s'agit alors d'un choix contraint. En outre, l'organisation du travail qu'elle ne manque pas de susciter, les impératifs liés au code du travail qu'elle implique, les objectifs de rentabilité ou de rationalisation des coûts qui la sous-tendent, sont autant de logiques qui viennent bien souvent percuter de plein fouet la dynamique propre à chaque personne handicapée et à son économie domestique. Des besoins sont évalués et pris en charge, des temps sont impartis, des forfaits sont attribués, des rythmes de vie sont imposés, et le " sur-mesure », le subjectif ou le personnalisé s'érodent face à l'organisation de l'intervention à domicile. Force est de constater que le projet de vie de la personne peut être totalement réaménagé par les conditions mêmes de sa mise en œuvre, et son désir noyé sous les flots d'une activité professionnelle agissant selon ses propres règles et principes. Ces prestations et services peuvent-ils exister sans gestion, planification et anticipation? Sans articulation desdites activités et des responsabilités ? Sans décision relative à un ordre qui permet à l'ensemble des acteurs de se coordonner au domicile de la personne ? Sans solliciter des expertises professionnelles ? De fait, l'intervention à domicile, de part les logiques qu'elle suppose, rend difficile la perpétuation d'une souveraineté sans partage de l'habitant sur son espace privé. C'est jusque dans les moindres détails d'une vie quotidienne, dans ce qui semble une banalité au professionnel parce que devenu routine à ses yeux, que se façonnent ces gestes essentiels à la vie quotidienne de la personne dépendante et que peut se perdre la maîtrise sur le domicile comme sur la vie personnelle.

Enfin, dernier paradoxe auquel doit faire face le professionnel : intervenir dans le cadre d'un ensemble de valeurs, de règles, de conventions et de responsabilités liées à sa profession sans pour autant porter atteinte à la sédimentation dont est construit le domicile de la personne soignée. De fait, comment faire avec un lit orienté en fonction du lever du soleil et non pas positionné au regard des va-et-vient des divers intervenants ? Comment ne pas déplacer nombre de petits objets qui semblent sans importance, pour les besoins du service ? Comment respecter le désordre ambiant, art de vivre pour certains, capharnaüm pour d'autres, alors qu'il en découle bien souvent une perte de temps pour l'intervention en cours ? Etc.

\section{Risque d'atteinte à la subjectivité réflexive}

Un dernier espace d'intimité est placé au cœur même de l'individu contemporain, un espace de subjectivité réflexive, largement promu par notre société individualiste. L'atteinte à ce type d'intimité est vécue par la personne, handicapée ou non, comme une atteinte à son être même, à ce qu'il y a de plus profond, de plus essentiel, de moins superficiel en elle. Et si elle ne sait généralement pas le définir ou le caractériser avec des mots, elle s'y identifie néanmoins très fortement. Cet espace réflexif est ce sur quoi est fondé le sentiment d'être son propre maître. De là découle le sentiment de s'appartenir ou encore la conviction d'être libre.

Bien évidemment, toute liberté est limitée et n'existe que dans un cadre de contraintes matérielles, sociales, légales, entre autres. II serait facile d'en conclure que, somme toute, chacun doit se confronter à ces restrictions, sans penser plus avant la question des causes des inégalités sociales et donc d'une certaine forme d'injustice. Or, entre les contraintes imposées et constituant la situation de dépendance, c'est-à-dire le traitement social et organisationnel de la personne dépendante, et les déterminismes restreignant la volonté de tout un chacun, un écart massif est constatable. Plusieurs raisons à cela.

Tout d'abord, les temps d'aide humaine sont évalués, forfaitisés, planifiés, minutés. Et tout ce qui n'est pas prévu ou anticipé, et par là-même partiellement financé, échappe à la personne, car, à quoi sert une volonté si elle n'a pas les moyens de ses aspirations ? Le handicap est souvent une longue attente après la disponibilité d'autrui. De plus, chaque acte de la vie quotidienne effectué par autrui est toujours quelque peu en écart avec le désir et la volonté de la personne dépendante, quelque soit la bonne volonté et les savoirs-faire de l'intervenant. En outre, un certain nombre de désirs ne sont que difficilement exprimables, soumis qu'ils sont au jugement ou bien aux tabous. Ces désirs, s'ils sont explicitement manifestés, peuvent être discutés, estimés comme en écart à la fiche de poste de l'intervenant, jugés inacceptables du point de vue de la bienséance, de la morale, de la civilité ou encore, autre argument imparable, de la santé. Ces désirs peuvent également être évalués comme ne relevant pas de besoins. Or, l'être humain se résume-t-il à ses besoins ? Tous ses besoins sont-ils vitaux? Précisément, l'être humain n'est-il pas Humain parce qu'il est bien 
davantage que des besoins?

Ainsi, cette subjectivité réflexive qui caractérise l'intimité de l'individu handicapé s'exprime dans un cadre au combien plus contraignant que ceux des valides, et pas seulement du fait d'une différence corporelle, mais bien aussi en raison de son traitement social. C'est pourquoi un enjeu politique majeur aujourd'hui est de penser davantage la compensation du handicap que le financement de la survie des personnes handicapées.

Par ailleurs, s'appartenir, être son propre maître, se gouverner selon ses propres règles, autrement-dit : être autonome en toute dépendance, suppose qu'à défaut de pouvoir effectuer par soi-même un certain nombre de gestes, il est tout du moins possible de décider et d'organiser sa vie par et pour soi-même. Or force est de constater qu'en raison de diverses contraintes légales, organisationnelles, professionnelles, etc., cette autonomie est aussi largement limitée dans sa volonté de tenter des expériences, de pendre quelques risques, et donc de se développer. De fait, les décisions des tutelles financières, les jeux organisationnels des institutions orientent bien souvent les disponibilités, les niveaux de qualification, les intervenants, les formations, les conditions de travail, etc., autant de facteurs contribuant à renforcer ou pas des dépendances. En outre, la question de la responsabilité professionnelle et institutionnelle engage couramment une réserve des mêmes intervenants vis-à-vis de toute prise de risque, laquelle est pourtant nécessaire notamment au développement d'une confiance en soi, d'une assurance. L'inconvénient qui en découle est donc un moindre épanouissement, une difficulté à déployer certains pans de son être, parfois même l'inhibition de facultés existantes ou le renoncement à choisir sa vie. L'atteinte à l'intimité s'exprime là par la restriction des espaces où être soi, l'invalidation d'une volonté d'autonomie en situation de dépendance.

Enfin, dernière atteinte à l'intimité comme subjectivité réflexive : les significations relatives à la dépendance transmises par de multiples canaux dans notre société aux individus, handicapés ou non. Cette habituation subtile au fil des jours à de nombreuses compréhensions dépréciatives des personnes en situation de handicap imprègne progressivement nos habitudes de pensée, construit nos a priori et influence nos comportements, que nous le voulions ou non. Nul n'est indemne des regards collectivement construits et portés sur des catégories d'individus. Et s'il fallait le démontrer, pensons à la curiosité et à l'intérêt suscités par un fauteuil roulant auprès d'enfants âgés de quelques années seulement, et, à la complexité relationnelle qu'il faudra assumer ensuite entre adultes valides et invalides au regard des peurs, jugements et inquiétudes de blesser réciproques qui sous-tendent à bas bruit les interactions. Quelle image de soi et de ses capacités, quels jugements sur elle-même peut élaborer une personne dépendante dans un environnement social plutôt disqualifiant? L'atteinte possiblement portée à la subjectivité dont il est ici question, est la construction et la transmission de la naturalité ou de la normalité de sa condition et de son traitement social. Cet effet de persuasion ou d'accoutumance à des évidences socialement portées au fil du quotidien, enraye tout proces d'élaboration d'une pensée alternative du handicap, laquelle doit toujours être envisagée comme possible. Par suite, la subjectivité exerce une autocensure sur elle-même. La volonté s'érode, l'espoir s'obscurcit, l'intellect et la créativité s'investissent systématiquement ailleurs que dans une pensée de la condition de l'être social dépendant.

\section{L'intimité : entre respect et liberté}

Cet éclairage porté sur la pénétration de l'intimité par nécessité met en lumière son fondement, son enrochement sur des paradoxes. Par conséquent, elle se révèle être une épreuve professionnelle et/ou personnelle, au sens où il s'agit de faire avec des injonctions contradictoires, voire incompatibles, en prenant sur soi la responsabilité de bricoler aux quotidiens des aménagements qui permettent à chacun, la personne dépendante et l'intervenant, de vivre au mieux les effets collatéraux. En effet, il est certainement impossible d'extirper totalement de ces activités leurs conséquences en matière d'assujettissement, de publicisation de la vie privée, d'impact sur la « sédimentation biographique » propre à chaque logement.

L'analyse succincte de ces quelques dimensions contemporaines de l'expérience de l'intimité permet de mettre en lumière un certain nombre de risques caractérisant la condition de l'être dépendant. L'ensemble de ces risques portent sur la possible atteinte à la personne par la restriction massive de sa liberté. C'est donc à la question du contrôle, de la maîtrise, de l'emprise sur l'individu dépendant à laquelle nous sommes confrontés. L'entrée dans l'intimité d'autrui par nécessité convoque des situations d'interaction atypiques, extra-ordinaires, et sur lesquelles il faudrait probablement réfléchir davantage, notamment en termes de conséquences sur les individus, professionnels ou usagers. Si cette entrée dans l'intimité par nécessite est 
utile et même indispensable, reste qu'elle n'est pas plus évidente, que naturelle ou normale. La pénétration de l'intimité par nécessité est construite de nombreux choix sociaux, portant aussi bien sur les conditions financières d'exercice des interventions, que sur leur organisation institutionnelle, sur la formation professionnelle des intervenants ou encore sur les modalités de compensation du handicap aujourd'hui soutenues, etc. Autrement-dit, d'autres alternatives, d'autres aménagements sont envisageables, et peut-être même souhaitables.

C'est assurément à ce endroit précis du raisonnement analytique, parce qu'il souligne la diversité des options dans l'exercice de la pénétration de l'intimité par nécessité, qu'il devient envisageable d'escompter trouver davantage de liberté pour l'ensemble des acteurs en jeu. En effet, promouvoir différentes modalités d'intervention a pour corollaire immédiat de soutenir la possibilité de choix entre diverses modalités d'intrusion dans son intimité pour la personne dépendante, et peut-être aussi le choix pour le professionnel de conditions de travail et de moyens avec lesquels il prévoit de soutenir cette épreuve professionnelle. A défaut de résoudre ces multiples paradoxes, introduire davantage de choix, soutenir une liberté accrue de décider, permet d'entendre et de prendre en considération la singularité du vécu de chacun face à ces risques interactionnels. Si l'épreuve est incontournable, elle peut être limitée dans ses conséquences par un environnement qui accorde du crédit aux ressentis exposés, de la valeur aux choix énoncés, qui accepte de se conduire avec réserve et retenue pour respecter autant qu'il est possible malgré une intrusion inéluctable.

C'est assurément dans un contexte sociétal sensible à la question de l'individu, à ses droits, ses devoirs et ses libertés, qu'une telle analyse trouve sa pertinence. Ces problèmes et ces paradoxes sont effectivement nouveaux, au sens où leur existence est étroitement liée à l'émergence d'une pensée du handicap et de la dépendance, non plus en termes de charité, d'assistance ou même de solidarité, mais en termes de droits humains et de droits civiques. Soulignons d'ailleurs à ce propos la mention de l'impératif de respecter l'intimité et la vie privée dans la loi de 2002 de rénovation de l'action sociale et médico-social. Rappelons l'existence d'un texte ratifié récemment par la France : la Convention relative aux Droits des Personnes Handicapées (ONU, 2006) qui encourage au respect de la dignité intrinsèque, de l'indépendance des personnes, de l'autonomie individuelle, y compris de la liberté de faire ses propres choix. Sans la promotion d'une telle vision de l'humain, les questions soulevées ici autour de la bientraitance, du care, et du respect, n'auraient pas de raisons d'être. L'ensemble de cet argument est donc à articuler avec l'apparition et le développement de nouvelles compréhensions de l'humain et de sa place dans la société. Un réaménagement de l'entendement concernant l'individu dépendant rend caduques différents problèmes et fonde dans le même temps des questions jusqu'alors non imaginées. C'est à une mutation des conceptions que nous assistons aujourd'hui, laquelle refonde et interroge le sens même des interventions à domicile. 